\title{
Tactile Suppression of Displacement
}

\author{
Mounia Ziat ${ }^{1}$, Vincent Hayward ${ }^{2}$, C. Elaine Chapman ${ }^{3}$, Marc O. Ernst $^{4}$ and Charles Lenay ${ }^{5}$ \\ ${ }^{l}$ Psychology Department, Wilfrid Laurier University, Waterloo, Canada \\ ${ }^{2}$ Institut des Systèmes Intelligents et de Robotique, UPMC Univ Paris 06, UMR 7222, Paris, France \\ ${ }^{3}$ Groupe de recherche sur le système nerveux central, École de réadaptation \& Département de Physiologie, Université de \\ Montréal, Montréal, Canada \\ ${ }^{4}$ Max-Planck-Institut für biologische Kybernetik, Tübingen, Germany \\ ${ }^{5}$ Technologie et Sciences de l'Homme, Université de technologie de Compiègne, Compiègne, France
}

Corresponding author: ziatmoun@cim.mcgill.ca

\begin{abstract}
In vision, the discovery of the phenomenon of saccadic suppression of displacement has made important contributions to the understanding of the stable world problem. Here we report a similar phenomenon in the tactile modality. When scanning a single Braille dot with two fingers of the same hand, participants were asked to decide whether the dot was stationary or whether it was displaced from one location to another. The stimulus was produced by refreshable Braille devices which have dots that can be swiftly raised and recessed. In some conditions the dot was stationary. In others, a displacement was created by monitoring the participant's finger position and by switching the dot activation when it was not touched by either finger. The dot displacement was of either $2.5 \mathrm{~mm}$ or $5 \mathrm{~mm}$. We found that in certain cases, displaced dots were felt to be stationary. If the displacement was orthogonal to the finger movements, tactile suppression occurred effectively when it was of $2.5 \mathrm{~mm}$, but when the displacement was of $5 \mathrm{~mm}$, the participants easily detected it. If the displacement was medial-lateral, the suppression effect occurred as well but less often when the apparent movement of the dot opposed the movement of the finger. In such cases, the stimulus appeared sooner than when the brain could predict it from finger movement, supporting a predictive rather than a postdictive differential processing hypothesis.
\end{abstract}

Keywords: tactile suppression effects, stable world problem, suppression of displacement, tactile perception

In all sensing modalities, individuals have the ability to experience a continuous and stable world in spite of the fact that stimulation changes constantly. The problem faced by the haptic system in order to provide perceptual stability is analogous to the problem faced by the visual system. In the two cases, the primary sensory input arises from sensitive surfaces that can be displaced motorically with respect to the outside world. As Poincaré pointed out (1897), self-generated movement has the benefit of enlarging the portion of the world that is accessible to the senses, but has the downside that it introduces a fundamental ambiguity: It is easy to construct stimuli that are identical when generated by one's own movements or due to the movement of an external agent. This problem has intrigued many scientists: Descartes, Purkinje, Helmholtz, Von Holtz and Mittelstaedt, Sperry, and others; see (Bays and Husain, 2003) for a brief review. More recently Wertheim (1994) and O'Regan \& Noe (2001) discussed the analogy between vision and touch from the viewpoint of perceptual stability. The general idea is that an organism needs to be aware of its own movements to resolve ambiguity and that assumptions must be made about the world, such as rigidity and immobility, to succeed at perceiving a stable world (Wexler et al., 2001). Some authors investigated other aspects of the somatosensory stability problem; one of them is object size perception in static contact with the skin for different body regions (Taylor-Clarke et al., 2004). Other studies contributed to identifying elements of stable tactile representations in the somatosensory cortex in the case of a passive hand (Fitzgerald et al., 2006; Haggard, 2006).

While surveying a scene, a specific instance of the stability problem is to decide that particular features of the external world remain at fixed locations, or conversely, that they move. This question is illustrated when considering the act of reading Braille. The Braille code is made of patterns of six closely packed raised dots. It is read by scanning lines of characters, often with two or more fingertips scanning over the same characters (Millar, 1997). Suppose that the leading finger reads the single dot of the letter "a". The nervous system must then decide, despite noise in the sensing and the motor systems, that it is the same dot that is read by the trailing finger. Of course, there should be strong prior assumption that the dot is immobile while being intangible between the two fingers, but the nervous system must nevertheless make a decision despite sensing and motor uncertainty. A similar problem arises when actively touching a feature with one finger and then returning to it with the same finger, but this case, the sensorimotor task is presumably subject to uncertainty to a lesser extent than in the two-finger case.

In vision, a well-known effect is the phenomenon of saccadic suppression of displacement (SSD), where a small change in the location of a target occurring during a saccade or during a blink is not consciously experienced (Bridgeman et al., 1975, Deubel 
et al., 2004). We therefore wondered whether a parallel effect occurs in touch since, like in vision, a small, fixed object in the world gives rise during scanning to a trajectory of sensory inputs distributed in time and space across the receptor surface. Haptic behavior may be compared to that of an eye fixating a target, losing the target due to a blink or a saccade to another location and then returning to it. In the single digit case, the analogy is appealing, yet cannot be very strict since there is no "peripheral touch" and since touch normally operates by scanning, not by fixating. In the two digit-case, the analogy could still be drawn but the return to the same target is effected by a different finger. It would be like having multiple fovea (Hatwell, 1986). Multi-digit haptic behavior could have points of comparison with peripheral vision.

In two experiments, we investigated what could be called tactile suppression of displacement, where a change in location of a stimulus scanned under two different fingertips (the index and the middle fingers in the present study) is not perceived during the gap between the two fingers; the stimulus, instead, is felt to be stationary. To evaluate this effect, we constructed an apparatus able to displace a tactile dot, that is, a small, yet easily detectable tactile feature, during the time interval beginning when the dot leaves the contact with one finger and ending when it makes contact again with the same or another finger.

As reported by Bridgeman et al. (1975), saccadic suppression of displacement yields a strong reduction in sensitivity (by three to four log units) in the detection of target's displacement during saccades when the displacement occurs shortly before or during a saccade. That a similar suppression of sensitivity occurs also around an eyeblink, not only during a saccade, suggests that similar processes might at play (Volkmann et al., 1980; Stevenson et al., 1986; Deubel et al., 2004). The interruption of sight during a blink is similar to the interruption of touch when a feature is in the gap between the two fingers during scanning.

\section{Related Effects}

To our knowledge, tactile suppression of displacement has not been described before, although several authors reported related effects. These effects are generally better described by stimulus suppression rather than relocation. In vision, flash suppression is an effect where a flash of light delivered during a saccade is not seen (Dodge, 1900; Wallach and Lewis, 1966; Matin, 1974). Tactile suppression has been reported to occur at different sites of the body when a weak electrical stimulus is applied. Results showed that the degree of inability to detect the presence of the stimuli during movement depends on its location (William et al, 1998), on intensity (William et al, 2000) and occurs during passive or active touch (Williams et al, 2002; Chapman and Beauchamp, 2006). Tactile suppression was also found for tactile stimuli moving laterally on the index for active and passive conditions (Vitello et al. 2006). In the active condition participants were asked to move their arm in a given direction while a tactile stimulus was applied to the fingertip. Results showed that there is a reduction of sensitivity during the active condition. This is in accordance with several other results that showed that tactile sensitivity decreases during movement (DyhrePoulson, 1978; Ghez and Lenzi 1971; Chapman, 1994).

Saccadic or flash suppression is quite distinct from a variety of other effects which can be classified under the collective name of change blindness (or change deafness, or even change numbness) whereby a change in a scene is not seen (or heard or felt) if that change occurs during a disruption (Rensink et al., 1997, Vitevitch, 2003, Gallace et al., 2006). In "change x-ness", the conditions are such that massive changes in the stimulation are blocked from reaching the conscious experience as the result of an attentional effect, eliciting considerable surprise once the participants are subsequently made aware of them. In contrast, with displacement suppression studied here, the change in the stimulation is limited to a small change of location of the stimulus in external coordinates and the attentional mechanisms are left operating undisturbed. The perceptual effect is in essence a spatial and temporal relocation of the stimulus, not a block and could be explained by a spatial remapping strategy analogous to what is described in vision (Bays and Husain, 2007).

\section{Overview of the present study}

We wondered whether touch exhibits suppression of displacement, presumably to facilitate the merging of peripheral inputs into a unified, stable percept. To test this hypothesis we designed two experiments where participants scanned a Braille dot that changed its location during the brief moment when not in contact with a finger during shifting from one finger to another, or during the moment needed for a single finger to return to the same target. In the two-finger trials, the Braille dot was scanned by the index and the middle fingertips (D2 and D3) of participants, but its position depended on whether D2 or D3 touched it. In the single-finger trials, the index digit (D2) scanned the dot and then returned to it. The stimuli were produced using a sensor sensitive to the fingers' position and connected to a computer that commanded the activation or the deactivation of the dots of a refreshable Braille cell. The setup ensured that the activation and deactivation of the dots occurred during the time interval beginning when the dot leaves contact with a finger and ending when it makes contact again. Because the dots of a refreshable Braille cell are $2.5 \mathrm{~mm}$ apart, this apparatus made it possible to program changes in location of $2.5 \mathrm{~mm}$ or of $5 \mathrm{~mm}$.

The dot spacing of Braille cells is so designed that dots are easily discriminated under static or dynamic touch. To establish that it was indeed the case, we asked 10 participants in a preliminary experiment under static touch to discriminate a displaced dot from a stationary 
using the Braille display used for the main experiments. The cell was programmed to activate and deactivate a single dot that sometimes reappeared at a different location $(2.5 \mathrm{~mm}$ or of $5 \mathrm{~mm}$ away in all four cardinal directions). All participants easily detected those changes, or lack thereof, and no significant effect nor any interactions due to direction or finger could be measured.

Throughout the main Experiment 1, using two fingers and active touch, the participants were asked to determine if they felt whether or not a dot was felt to be at the same location on the device. There were cases when the dot remained fixed at various positions, cases when it was displaced upward by one space (distal displacement relative to the long axis of the finger), and cases when it was displaced downward (proximal) by one space. In other cases the dot was displaced upward or downward by two spaces. In yet other cases it was displaced laterally, left or right, by one or two spaces. We also performed a control experiment, Experiment 2, with the same materials, method and task, but instead of scanning the same target with two fingers, the participants scanned the target with one single finger and then scanned the target in the opposite direction immediately after. Experiment 2 enabled us to verify that tactile suppression of displacement is not due to finger motion but to the brief interruption of sensory input when a feature is in the gap between two fingers.

\section{General Materials and Methods}

\section{Experiment 1}

\section{Participants}

Thirty McGill University students (12 males and 18 females) took part in Experiment 1. Twenty six of them declared that they were right handed and four that they were left handed. The participants' mean age was 25 (range 20-30). All reported a normal sense of touch and were not aware of any motor or cutaneous sensation deficits. They were naive as to the purpose of the experiments and they were paid for their participation. The institutional ethics committee approved the experimental protocol, and participants gave their informed consent before participating.

\section{Apparatus}

The apparatus employed commercially available refreshable Braille cells (Model B11, Metec-AG, Stuttgart, Germany), see Figure 1, embedded in a jig designed to establish a repeatable relationship between the fingers and the target region. A refreshable Braille cell is a computer-controlled device with two columns of four dots (they have an extra row compared to paper Braille) that are actuated to recess rapidly inside cavities, or to protrude out of the surface. This jig guided the participants' fingers to scan the target surface in straight line, lateral sweeps.

Referring to Figure 1, the apparatus comprised two refreshable Braille-cells with eight nearly hemispherical dots each (radius, $0.7 \mathrm{~mm}$; height, $0.7 \mathrm{~mm}$ ) permitting a great variety of tactile patterns to be displayed and a two-finger cradle supported by a sliding potentiometer that could be adjusted on a Velcro surface to accommodate the participants' anatomical differences. The cells were recessed in a slightly inclined surface and positioned so as to stimulate participants' fingertips and to make sure that both fingertips were in contact with the tactile display. Each cell measured $6.42 \times 17.8 \mathrm{~mm}$ with a dot spacing of $2.5 \mathrm{~mm}$. A computer controlled the activation of the dots according to the potentiometers readings (slider $60 \mathrm{~mm}$, Phidgets, Inc., Calgary, Canada).

The apparatus was hidden from the view of the participants by a screen. They slid their hands through an opening to reach the target surface, guided initially by the experimenter. A computer monitor was used to give instructions and feedback regarding the finger position. The index and the middle fingers do not have the same length. Because of this difference, the fingers grazed the target surface at slightly different regions on each fingerpad. The cradle position was adjusted to ensure that both fingerpads were in contact with the cells in a relaxed position, taking into account the different lengths of the fingers. A relaxed position was achieved when adopting a slightly bent finger posture.
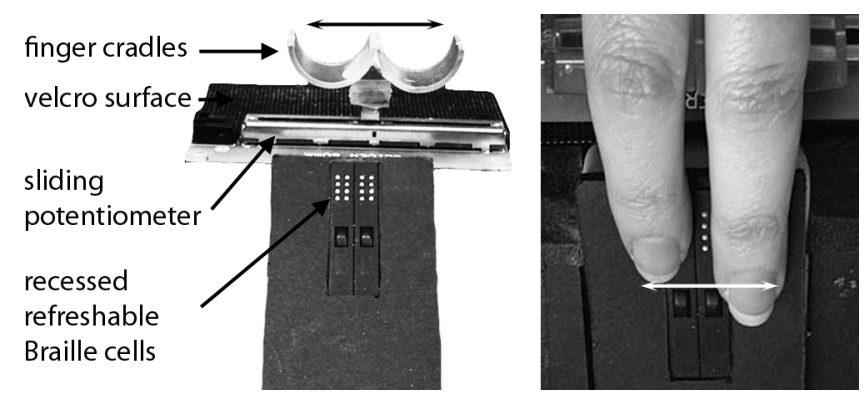

Fig. 1 Apparatus. Refreshable Braille cells are embedded in a inclined surface (left). A sliding potentiometer supports two semicylindrical cradles forming a guide that can be relocated on a Velcro surface to accommodate the participants' anatomical differences. Two fingers (D2 \& D3), seen from the top, scan laterally the target surface comprising an array of Braille dots [2× $(2 \times 4)]$. The dots could be swiftly lifted or recessed into the surface, under computer control.

\section{Stimuli}

The sixteen dot patterns used in the Experiment 1 are listed in Table 1. Each pattern in the displacement condition (D) has an equivalent in the stationary condition (S). The patterns were created systematically by causing the dot to change location in the four cardinal directions by one or by two spaces. These directions are termed proximal, distal, medial, and lateral when discussed in relation with anatomical coordinates; and down, up, left, and right respectively when describing the position of the dots on the display. For purposes of illustration, Figure 2 shows two examples of how two 
digits, D2 and D3, can experience a dot change. When scanning pattern 1 (Figure 2a) starting from the right, digit D2 first scans over a raised dot (black circle, grey when hidden), then, while in the gap, the dot is recessed (white circle) and another is raised. For pattern 9 (Figure $2 \mathrm{~b}$ ) the dot is displaced to the right by recessing it and raising it at another location, while in the gap. When scanning occurs in the opposite direction, the sequence of events is reversed. The figure is not to scale for clarity. In practice the dots were much smaller, so there was sufficient room for the dots to change location laterally in the gap without being in contact with either finger. a
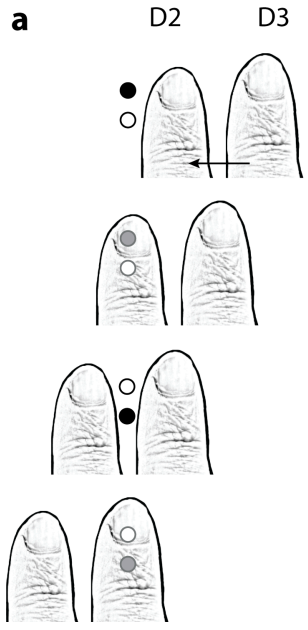

b
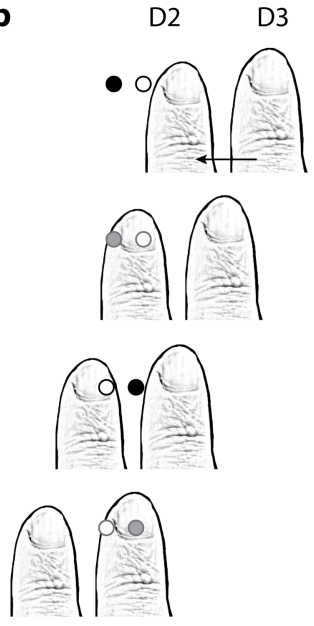

Fig. 2 Two fingers, D2 and D3, scan the target surface, from right to left. (a) If pattern 1 was presented, D2 met the raised dot (black then grey) in the upper position, then scans it. When in the gap between the two fingers, the dot was switched one place down so that D3 scanned it at a different location. (b) If pattern 9 was presented, D2 met the raised dot in the left position, then scanned it. When in the gap between the two fingers, the dot was switched one place right so that D3 scanned it as if it moved in a direction opposite to the movement of the fingers.

\section{Design}

The experimental design was completely symmetrical and counterbalanced. Experiment 1 comprised two blocks of 80 trials. In each block, a randomized mix of displaced or stationary dots was presented in equal proportions. Each block of trials lasted about 10 minutes and was followed by a short break of 3 minutes. In condition S (stationary), the same dot was scanned by the index and by the middle fingertip. In condition $\mathrm{D}$ (displacement), the dot changed location when not in contact. In all cases the total duration of the trial was 5 seconds. In each block the 16 different patterns ( 8 with displacement and 8 stationary) were therefore presented five times each (ten times for all the experiment).
Table 1

The participants were tested with sixteen combinations of dot positions tabulated below. A $\bullet$ represents a raised dot and a $\bigcirc$ represents a recessed dot. Patterns corresponding to a dot apparent displaced are collected in the left column (Condition D). In the right column (Condition S), the counterpart patterns where the dot remained at a fixed location are shown.

\begin{tabular}{|c|c|c|c|c|c|}
\hline \multicolumn{3}{|c|}{ Condition D } & \multicolumn{3}{|c|}{ Condition $\mathrm{S}$} \\
\hline $\begin{array}{l}\text { Pat. } \\
\text { num. }\end{array}$ & D2 & D3 & $\begin{array}{l}\text { Pat. } \\
\text { num. }\end{array}$ & D2 & D3 \\
\hline 1 & $\begin{array}{l}0000 \\
0000 \\
0000 \\
0000\end{array}$ & $\begin{array}{l}0000 \\
0000 \\
0000 \\
0000\end{array}$ & 2 & $\begin{array}{l}0000 \\
0000 \\
0000 \\
0000\end{array}$ & $\begin{array}{l}0000 \\
0000 \\
0000 \\
0000 \\
\end{array}$ \\
\hline 3 & $\begin{array}{l}0000 \\
0000 \\
0000 \\
0000\end{array}$ & $\begin{array}{l}0000 \\
0000 \\
0000 \\
0000\end{array}$ & 4 & $\begin{array}{l}0000 \\
0000 \\
0000 \\
0000\end{array}$ & $\begin{array}{l}0000 \\
0000 \\
0000 \\
0000 \\
\end{array}$ \\
\hline 5 & $\begin{array}{l}0000 \\
0000 \\
0000 \\
0000\end{array}$ & $\begin{array}{l}0000 \\
0000 \\
0000 \\
0000\end{array}$ & 6 & $\begin{array}{l}0000 \\
0000 \\
0000 \\
0000\end{array}$ & $\begin{array}{l}0000 \\
0000 \\
0000 \\
0000 \\
\end{array}$ \\
\hline 7 & $\begin{array}{l}0000 \\
0000 \\
0000 \\
0000\end{array}$ & $\begin{array}{l}0000 \\
0000 \\
0000 \\
0000\end{array}$ & 8 & $\begin{array}{l}0000 \\
0000 \\
0000 \\
0000\end{array}$ & $\begin{array}{l}0000 \\
0000 \\
0000 \\
0000 \\
\end{array}$ \\
\hline 9 & $\begin{array}{l}0000 \\
0000 \\
0000 \\
0000\end{array}$ & $\begin{array}{l}0000 \\
0000 \\
0000 \\
0000\end{array}$ & 10 & $\begin{array}{l}0000 \\
0000 \\
0000 \\
0000\end{array}$ & $\begin{array}{l}0000 \\
0000 \\
0000 \\
0000 \\
\end{array}$ \\
\hline 11 & $\begin{array}{l}0000 \\
0000 \\
0000 \\
0000\end{array}$ & $\begin{array}{l}0000 \\
0000 \\
0000 \\
0000\end{array}$ & 12 & $\begin{array}{l}0000 \\
0000 \\
0000 \\
0000\end{array}$ & $\begin{array}{l}0000 \\
0000 \\
0000 \\
0000 \\
\end{array}$ \\
\hline 13 & $\begin{array}{l}0000 \\
0000 \\
0000 \\
0000\end{array}$ & $\begin{array}{l}0000 \\
0000 \\
0000 \\
0000\end{array}$ & 14 & $\begin{array}{l}0000 \\
0000 \\
0000 \\
0000\end{array}$ & $\begin{array}{l}0000 \\
0000 \\
0000 \\
0000 \\
\end{array}$ \\
\hline 15 & $\begin{array}{l}0000 \\
0000 \\
0000 \\
0000\end{array}$ & $\begin{array}{l}0000 \\
0000 \\
0000 \\
0000\end{array}$ & 16 & $\begin{array}{l}0000 \\
0000 \\
0000 \\
0000\end{array}$ & $\begin{array}{l}0000 \\
0000 \\
0000 \\
0000 \\
\end{array}$ \\
\hline
\end{tabular}

\section{Procedure}

The participants were seated comfortably in front of a table. They wore earphones to block unwanted auditory cues. Both forearms were supported by armrests. The apparatus was positioned in front of the dominant arm in a comfortable position so that the fingertips were at rest when grazing the target surface and it was hidden from view by a screen. The participants had their middle and index fingers supported by the cradle that guided their movements. They scanned the target with both their index and middle fingers, always starting with the index. In all experiments, they scanned the surface laterally twice back and forth starting from the right if they were right handed, for a distance of $60 \mathrm{~mm}$, which corresponded to the surrounding context. Left-handed participants started from the left and right-handed participants from the right in order to preserve an identical movement-finger relationship (pattern 11 for the left-handed corresponded to pattern 9 for the righthanded, and vice-versa, see Table 1). The participants 
were instructed to press one of two keys marked "same" (location) and "not same" (location) on a computer keyboard with their non-dominant hand as soon as they decided whether or not a change in location was felt. The trial was terminated if no response was made within 5 seconds. They pressed the enter key to start the next trial. No feedback was given regarding the participants response. At the beginning of the experiment, 20 practice trials (10 moving and 10 stationary dots in random order) were administered for the participants to become familiar with the device and the stimuli; feedback on performance was given for these practice trials and also they could also estimate the speed of their movements, which was approximately $48 \mathrm{~mm} / \mathrm{s}$. The scanning rate was similar between participants since they covered the $60 \mathrm{~mm}$ span twice back and forth in $5 \mathrm{~s}$ consistently before giving an answer.

\section{Experiment 2}

\section{Participants}

Ten Wilfrid Laurier University students (4 males and 6 females) took part Experiment 2. All declared that they were right handed. The participants' mean age was 25 (range 20-30). All reported a normal sense of touch and were not aware of any motor or cutaneous sensation deficits. They were naive as to the purpose of the experiments and they were paid for their participation. The institutional ethics committee approved the experimental protocol, and participants gave their informed consent before participating.

\section{Stimuli}

The sixteen dot patterns used in Experiment 2 are the same one used in Experiment 1 and are listed in Table 1. In Experiment 2, only one finger is used. For example, when scanning pattern 1 starting from the right, digit D2 scanned over a raised dot, then, when D2 scanned the display in the opposite direction, pattern 2 was displayed. The displacement always occurred while the finger was not touching the display.

\section{Design}

The experimental design was completely symmetrical and counterbalanced. Experiment 2 consisted of 40 trials per block. In each block, a randomized mix of moving or stationary dots was presented in equal proportions. Each block of trials lasted about 5 minutes and was followed by a short break of 3 minutes. In condition $S$, the same dot was scanned by the index finger. In condition $\mathrm{D}$, the dot changed location when not in contact. In all cases the total duration of the trial was 5 seconds. In all the experiment, the 16 different patterns $(8$ moving and 8 stationary) were therefore presented five times each.

\section{Procedure}

The same apparatus as in Experiment 1 was used and the same procedure was followed. The participants were seated in front of a table and the apparatus, hidden by a screen and positioned in front of the right hand. The participants had their middle and index fingers supported by the cradle that guided their movements but they scanned the target with their index finger only. The participants had to press one of two keys (similarly marked "same" and "not same") on a computer keyboard as soon as they decided whether or not a change in location on the device was felt. The trial was terminated if no response was made within 5 seconds. At the beginning of the experiment, 20 practice trials (10 displaced and 10 stationary dots in random order) were administered for the participants to become familiar with the device and the stimuli.

\section{Measures}

A three-way repeated measures ANOVA was used to assess the effects of conditions (stationary, displacement), amplitude $(2.5 \mathrm{~mm}, 5 \mathrm{~mm})$ and direction (up, down, left, right) on participants' performances. According to whether interaction effects were significant or not, we conducted marginal pairwise or simple effect tests. Simple effects were analyzed using the one-way repeated measures ANOVA on each subset of the data. Pairwise comparisons were conducted using dependent samples $t$-test. Finally, the Fisher Exact Test was used to examine the significance of the associations between the two experiments for each stimulus because it is well suited for small sample sizes and compares the associations between two variables.

\section{Results}

\section{Experiment 1}

The hypothesis was that under certain conditions (type and amplitude of displacement) participants would fail to detect that dots changed location between their fingers, thus suppressing tactile stimulus displacement. A preliminary experiment demonstrated that theses displacements were well above detection threshold. We expected that the distance between the dots and the direction of motion relative to the fingers would be important factors. A displacement of $2.5 \mathrm{~mm}$ should be more suppressed than one of $5 \mathrm{~mm}$ and we also tested whether the direction of motion played a role in tactile suppression of displacement.

Among the thirty participants, the results of one individual were dropped from all analyses because he could not perform the task. In the remaining 2320 trials, 46 trials were removed from the analysis on the basis that no movement was detected. We verified that the scanning rate values for the remaining trials were close to $48 \mathrm{~mm} / \mathrm{s}$.

\section{Correct rate for stationary dots and incorrect rate for moving dots.}

We performed a three-way ANOVA repeated measures within the three factors: amplitude, direction and condition. We were primarily interested in the incorrect rate for the change condition which was when a dot displaced between the fingers but was judged to be stationary. For comparison purpose, we also determined 
the incorrect rate for the stationary condition, which was a stationary dot that was judged to have displaced. The displacement suppression occurred when the subjects were not able to discriminate a stationary dot from a displaced dot. All effects are reported to be significant for $p<.05$.

There was a significant effect of the condition, $F(1,28)=13.66$. Contrasts revealed that the incorrect rate for displaced dots was higher than for the stationary dots, $r=0.57$. There was also a significant main effect of the amplitude, $F(1,28)=39.31$. Contrasts showed that the incorrect rate for the $2.5 \mathrm{~mm}$ amplitude was higher than for the $5 \mathrm{~mm}$ amplitude, $r=0.76$. There was a significant effect of the direction, $F(3,84)=4.32$. Contrasts showed that the incorrect rate for the right direction was higher than for the down direction, $F(1,28)=8.22, r=0.48$ and for the left direction, $F(1,28)=8.96, r=0.49$ directions.

There was significant interaction between the condition and the amplitude factors, $F(1,28)=18.29$. Contrasts revealed that the incorrect rates were higher when the dot was displaced with a small amplitude than when the dot was stationary, $r=0.63$. The incorrect rates were similar for both conditions for the $5 \mathrm{~mm}$ amplitude.

There was significant interaction between the condition and the direction factors, $F(3,84)=4.55$. Contrasts were used to compare the effect of condition across the four directions levels. The results showed that for the stationary condition, the incorrect rate for the right direction was higher than for the up direction, $F(1,28)=11.37, \quad r=0.54$, for the down direction, $F(1,28)=5.60, r=0.41$, and for the left direction, $F(1,28)=6.20, r=0.43$.

There was significant interaction between the amplitude and the direction factors, $F(1,28)=5.88$. Contrasts revealed that for the stationary condition, the incorrect rate for the right direction was higher than for the up direction $F(1,28)=9.20, r=0.50$, the down direction, $F(1,28)=7.19, r=0.45$ and the left direction, $F(1,28)=11.44, r=0.54$.

Finally, the condition $\times$ amplitude $\times$ direction interaction was significant, $F(3,84)=3.97$. This finding indicates that the amplitude $\times$ direction interaction described previously was different in the displacement and stationary conditions. Again, contrasts were used to break up the interaction; they compared incorrect rates for the two conditions for each level of amplitude across each level of direction. The first two contrasts looked at differences between conditions, comparing amplitudes for the up versus right directions, and for left versus right directions. The contrasts were significant for up versus right directions, $F(1,28)=4.65, r=0.38$ and for left versus right directions, $F(1,28)=7.89, r=0.47$. For the stationary condition, regardless of amplitude, the incorrect rate for the right direction was higher than for the up and left directions.

For the displacement condition, while the incorrect rate is the same for the two amplitudes in the right direction, the incorrect rate decreased for the $5 \mathrm{~mm}$ amplitude and increased for the $2.5 \mathrm{~mm}$ amplitude, both for the up and left directions. A contrast compared the down direction to the left direction when the $2.5 \mathrm{~mm}$ amplitude was compared to the $5 \mathrm{~mm}$ amplitude and when the displacement condition was compared to the stationary condition. This contrast was significant, $F(1,28)=5.94, r=0.42$ and revealed that for the two amplitudes the incorrect rate was higher for the displacement condition than for the stationary condition, except for the left direction with a $5 \mathrm{~mm}$ amplitude.

To verify the interpretation of the interaction effects and these contrasts, we conducted simple effect tests using one-way repeated measures ANOVA on each subset of the data. For clarity, Table 2 summarized the correspondence between and each of each pair of factors and stimulus number. Pairwise comparisons were conducted using the dependent samples $t$-test on each pair of factors. The results are collected in Table 3 for each factor.

Table 2

Summary of each pair of factors (condition, amplitude and direction).

Cond.

Displacement

\begin{tabular}{lcccccccc}
\cline { 2 - 9 } Amp. & \multicolumn{4}{c}{$2.5 \mathrm{~mm}$} & \multicolumn{5}{c}{$5 \mathrm{~mm}$} \\
nir. & up & dwn & left & right & up & dwn & left & right \\
Pat. & 1 & 3 & 9 & 11 & 5 & 7 & 13 & 15 \\
& & & & & Stationary & & & \\
& & & & & & & & \\
& up & dwn & left & right & up & dwn & left & right \\
& 2 & 4 & 10 & 12 & 6 & 8 & 14 & 16
\end{tabular}

Table 3

Summary of the results of Experiment 1 from 29 subjects, giving one-way ANOVA $F$ and $p$ values for the condition, amplitude factors.

\begin{tabular}{ccll}
\hline Factor & Pattern pairs & $(\mathrm{F}, \mathrm{d})$ & $\mathrm{p}$ \\
\hline Condition & 1 vs. 2 & $(20.96,28)$ & $0.0001^{*}$ \\
& 3 vs. 4 & $(9.16,28)$ & $0.005^{*}$ \\
5 vs. 6 & $(0.72,28)$ & 0.40 \\
& 7 vs. 8 & $(2.92,28)$ & 0.10 \\
& 9 vs. 10 & $(36.57,28)$ & $0.0001^{*}$ \\
& 11 vs. 12 & $(0.001,28)$ & 0.97 \\
& 13 vs. 14 & $(0.20,28)$ & 0.66 \\
& 15 vs. 16 & $(0.1,28)$ & 0.76 \\
\hline Amplitude & 1 vs. 5 & $(30.89,28)$ & $0.0001^{*}$ \\
& 3 vs. 7 & $(8.45,28)$ & $0.007^{*}$ \\
& 4 vs. 8 & $(6.48,28)$ & $0.017^{*}$ \\
& 9 vs. 13 & $(37.83,28)$ & $0.0001^{*}$ \\
\hline
\end{tabular}


The $t$-tests for the direction factor indicated that the incorrect rates associated with patterns 1 and 3 ( $2.5 \mathrm{~mm}$ changes) were significantly different $(p<0.01)$ from each other, and from the other patterns $(5,7$ and 11). In contrast, the incorrect rates associated with patterns 5 and 7 ( $5 \mathrm{~mm}$ changes) were similar, and lower than for the smaller displacements. The $t$-tests for the rightward-leftward displacements also indicated that the incorrect rates associated with patterns 9 and 13 (rightward changes) were significantly different $(p<0.01)$ from each other, and the other patterns $(11,15)$. In contrast, the incorrect rates associated with patterns 11 and 15 (leftward displacements) were similar, and lower than for the rightward displacements (see Figure 3). Incorrect rates depended on the distance and the direction of the dot displacement; they were high for pattern 1, 3 and 9 when the distance between dots is small and for pattern 11 and 15 when dots were displaced from right to left.

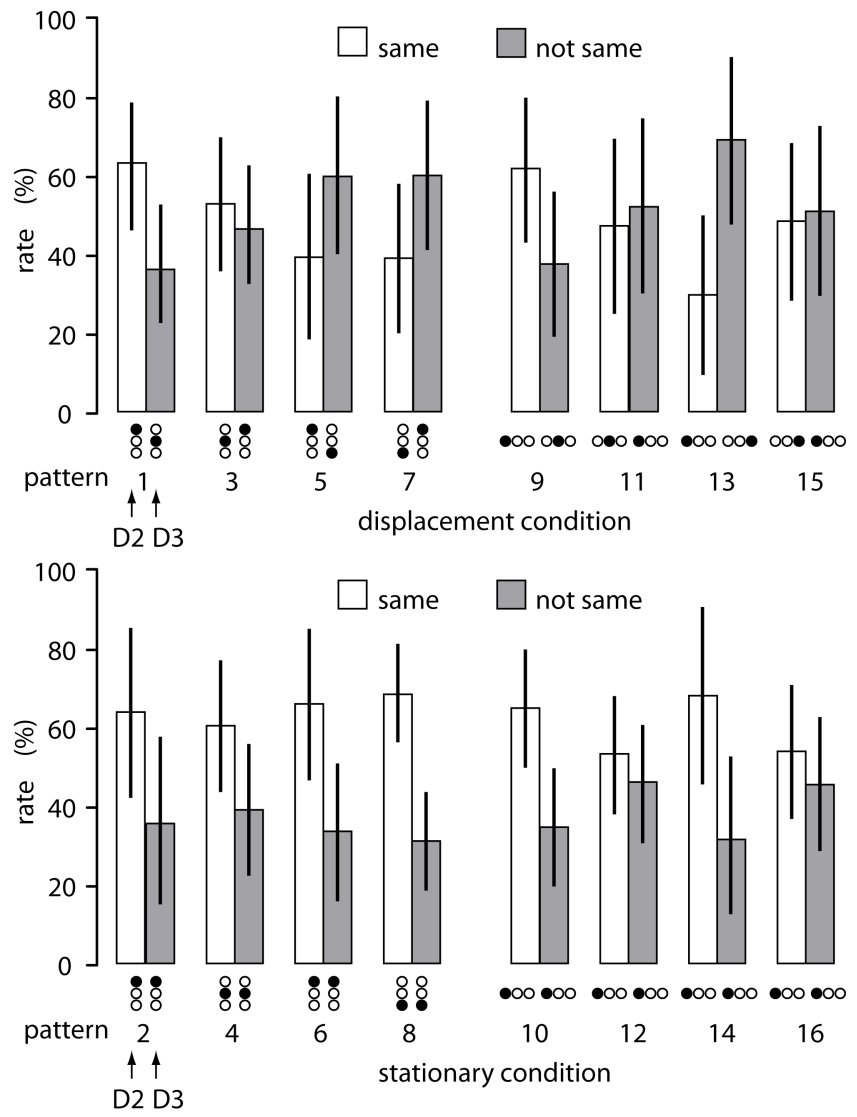

Fig. 3 Participants' responses for (+/- sd) for proximaldistal and medial-lateral patterns for Experiment 1. The results are sorted by pattern types (proximal-distal: left column; medial-lateral: right column; displacement condition top row; stationary condition bottom row).

In addition to the contrasts tests performed above, $t$-tests also indicated that the incorrect rates associated with patterns 12 and 16 (stationary condition corresponding to leftward displacements) were significantly different (Table 4) from the other patterns
$(2,4,10$, and 14) and $(6,8,10$, and 14), respectively, which means that participants answered more often "not same" for the static pattern corresponding to the leftward displacements.

Table 4

Summary of the results of Experiment 1 from 29 subjects, giving paired $t$-test and $p$ values for the direction factors (only the significant effects are displayed); Proximal and distal displacements are shown under the direction factor.

\begin{tabular}{ccll}
\hline Factor & Pattern pairs & $(\mathrm{t}, \mathrm{d})$ & $\mathrm{p}$ \\
\hline Direction & 1 vs. 3 & $(3.06,28)$ & $0.005^{*}$ \\
& 1 vs. 11 & $(4.01,28)$ & $0.001^{*}$ \\
3 vs. 11 & $(2.02,28)$ & $0.053^{*}$ \\
9 vs. 11 & $(2.95,28)$ & $0.006^{*}$ \\
2 vs. 12 & $(2.44,28)$ & $0.021^{*}$ \\
& 4 vs. 12 & $(2.32,28)$ & $0.028^{*}$ \\
10 vs. 12 & $(3.32,28)$ & $0.003^{*}$ \\
13 vs. 15 & $(3.06,28)$ & $0.005^{*}$ \\
6 vs. 16 & $(2.71,28)$ & $0.011^{*}$ \\
8 vs. 16 & $(3.95,28)$ & $0.0001^{*}$ \\
14 vs. 16 & $(2.72,28)$ & $0.011^{*}$ \\
1 vs. 7 & $(5.92,28)$ & $0.0001^{*}$ \\
3 vs. 5 & $(3.34,28)$ & $0.002^{*}$ \\
9 vs. 15 & $(2.88,28)$ & $0.007^{*}$ \\
11 vs. 13 & $(3.20,28)$ & $0.003^{*}$ \\
10 vs. 16 & $(2.77,28)$ & $0.01^{*}$ \\
12 vs. 14 & $(3.95,28)$ & $0.0001^{*}$ \\
\hline
\end{tabular}

Finally, for the condition factor, as shown in Table 3, the one-way ANOVA revealed a significant effect for patterns 1 vs. 2,3 vs. 4 , and 9 vs. 10, which corresponded to $2.5 \mathrm{~mm}$ displacements downward, upward, rightward where the incorrect rates were significantly higher for the displaced dots than for stationary ones. All the other patterns, 5 vs. 6,7 vs. 8 , 11 vs. 12,13 vs. 14 and 15 vs. 16 , corresponding to a proximal-distal $5 \mathrm{~mm}$ downward, a $5 \mathrm{~mm}$ upward, a 2.5 $\mathrm{mm}$ leftward, a $5 \mathrm{~mm}$ leftward and a $5 \mathrm{~mm}$ rightward displacements, respectively had the same incorrect rates for stationary dots. The rate of incorrect judgments for displaced dots increased for $2.5 \mathrm{~mm}$ changes in mediallateral and rightward directions but not for leftward direction. The one-way ANOVA for the amplitude factor confirmed this prediction since a significant effect was noticed for the patterns 1 vs. 5, 3 vs. 7 and 9 vs. 13, which correspond to 2.5 and $5 \mathrm{~mm}$ changes in location downward, upward, and rightward, respectively. We also noticed a significant effect for the pattern 4 vs. 8 that are stationary. It also confirms contrast tests that showed that the higher incorrect rates were obtained for right direction for both stationary and displaced dots 
Authors' copy accepted to Experimental Brain Research, 2010.

Subjects did not perceive the smallest displacements, regardless of the direction (proximaldistal (patterns 1 and 3); medial-lateral (patterns 9 and 11) but also did not perceive stationary dots for leftward direction (patterns 12 and 16). In contrast, the larger $5 \mathrm{~mm}$ displacements in the proximal-distal direction (patterns 5 and 7) were perceived. The results were less clear-cut for the medial-lateral $5 \mathrm{~mm}$ changes: leftward changes in location (pattern 13) were perceived while rightward changes (pattern 15) were not.

\section{Experiment 2}

Here, we expected that the participants were able to detect without any difficulty the difference between the two conditions $\mathrm{D}$ and $\mathrm{S}$.

A three-way repeated measures ANOVA with the three factors condition (D or S), amplitude $(2.5 \mathrm{~mm}$, $5 \mathrm{~mm}$ ) and direction (left, right, up, down) was used to assess participants' incorrect rate. Interactions of combinations of factors taken two by two were not significant. The interaction of the three factors was not significant. There were also no significance effects for the factors amplitude, $F(1,9)=3.72, p=.09$, direction, $F(3,27)=.12, \quad p=.96$, and condition, $F(1,9)=.22$, $p=.65$.

Table 5 displays participants' correct and incorrect rates for each stimulus. As expected, participants were able to make the difference between a dot that was stationary and a dot that was moving while scanning Braille cells with one single finger.

\section{Table 5}

Summary of the results of Experiment 2 from 9 participants, giving the mean of correct $(\mathrm{C})$ and incorrect rates (IC) for each condition, paired $t$-test and $p$ values.

\begin{tabular}{ccccc}
\hline & \multicolumn{2}{c}{ Displacement condition } & \multicolumn{2}{c}{ Stationary condition } \\
\hline $\begin{array}{l}\text { Pattern } \\
\text { pairs }\end{array}$ & $\begin{array}{c}\text { \% of IC } \\
\text { odd stim. }\end{array}$ & $\begin{array}{c}\text { \% of C } \\
\text { odd stim. }\end{array}$ & $\begin{array}{c}\text { \% of IC } \\
\text { even stim. }\end{array}$ & $\begin{array}{c}\text { \% of C } \\
\text { even stim. }\end{array}$ \\
\hline 1 vs. 2 & 38.00 & 62.00 & 22.00 & 78.00 \\
3 vs. 4 & 30.00 & 70.00 & 33.00 & 67.00 \\
5 vs. 6 & 21.00 & 79.00 & 29.00 & 71.00 \\
7 vs. 8 & 15.00 & 85.00 & 26.00 & 74.00 \\
9 vs. 10 & 41.34 & 58.66 & 23.00 & 77.00 \\
11 vs. 12 & 22.00 & 78.00 & 31.09 & 68.91 \\
13 vs. 14 & 22.00 & 78.00 & 25.00 & 75.00 \\
15 vs. 16 & 25.00 & 75.00 & 19.00 & 81.00 \\
\hline
\end{tabular}

The results of Experiment 2 were very different from those of Experiment 1 where some patterns are suppressed during the scan and others not. Table 6 shows the results of the Fisher's Exact Test that was used to examine the significance of the association between the experiments. If the direction of a displacement has no effect during one-finger scanning, it does when scanning with two fingers.
Final version Vol 206, No 3, pp. 299-310

Table 6

Percentage of correct answers $(\mathrm{C}=$ number of "same" judgments for stationary dots + number of "not same" judgments for moving dots) for the two experiments and for each pair of stimuli. Results of Fishers Exact Test for each experiment and for each pair of stimuli.

\begin{tabular}{cccc}
\hline Pattern & Exp. 1 & Exp 2 & Exp. 1- Exp 2 \\
\hline & C & C & Fisher's Exact \\
\hline 1 vs. 2 & 50,34 & 71,00 & 0.0001 \\
3 vs. 4 & 53,79 & 71,00 & 0.001 \\
5 vs. 6 & 63,34 & $\overline{79,00}$ & 0.002 \\
7 vs. 8 & $\mathbf{6 4 , 6 6}$ & $\overline{85,00}$ & 0.0001 \\
9 vs. 10 & $5 \overline{1}, \overline{55}$ & $69, \overline{8}$ & 0.001 \\
11 vs. 12 & $5 \overline{3}, 69$ & $7 \overline{7}, 11$ & 0.0001 \\
13 vs. 14 & $\mathbf{6 9 , 1 4}$ & 80,00 & 0.03 \\
15 vs. 16 & $5 \overline{3}, 10$ & $8 \overline{1}, 00$ & 0.0001 \\
\hline
\end{tabular}

\section{Discussion}

These experiments were designed to confirm the existence of the tactile suppression of displacement phenomenon, which is for touch, what saccadic suppression of displacement is for vision. The results showed that subjects could not perceive small $(2.5 \mathrm{~mm})$ displacements, although they could perceive most of the larger displacements. There was a significant effect due to the size of the displacement, which because of the nature of the stimulus delivery method had only two values, 2.5 and $5 \mathrm{~mm}$. This result confirms the existence of displacement suppression when the amplitude is small enough; yet well above the two-point detection threshold and the spatial acuity for passive and active touch, which is about $1.5 \mathrm{~mm}$ (Heller, 1986; Loomis, 1985; Craig, 1999). Some large displacements were less suppressed than others and suppression rates were significantly higher when the dots were displaced leftward, which merits discussion.

\section{Direction of the displacement}

First let us consider displacements in the proximal-distal direction. Recall that fingers have different lengths. As a result, when the dots were displaced downward, they remained substantially within the central portion of each fingerpad. On the other hand when the dots were displaced upward, they systematically shifted from the central portion of the D2 fingerpad to the lower portion of the D3 fingerpad (see Figure 4). Craig showed that the tactile spatial acuity is uniform over the distal $2 / 3$ of the distal phalanx (glabrous skin), but rises proximally (Craig, 1999). This inhomogeneity can be attributed to the non-uniform innervation density of the distal regions of the fingers (Craig 1999; Johnson et al., 1981). It may be speculated that this non-uniform distribution is also reflected in the somatotopic cortical representations of tactile stimuli. In our experiment, suppression occurred for all single-space proximal-distal displacements, but 
when dots were displaced distally, the stimulus crossed the boundary from the less sensitive portion of the fingertip to the more sensitive region which may explain the different result obtained from patterns 1 and 3 .
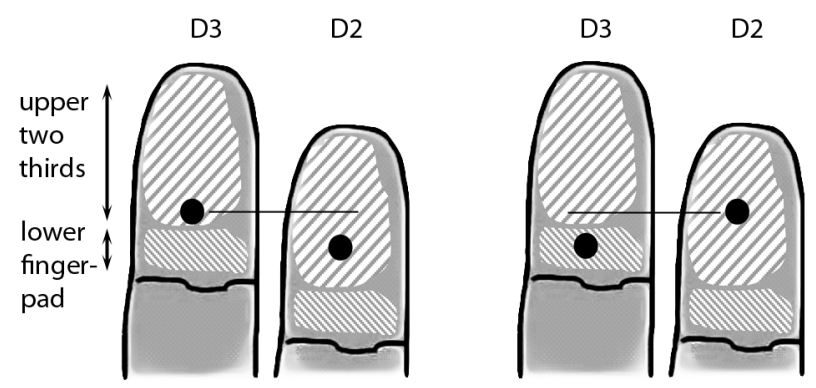

Fig. 4 When the dot was displaced downward it remained in the central portion of the fingerpads but when it was displaced upward it shifted from the lower portion of D3 to the central portion of D2. The figure shows the volar surface of the fingers and the areas are in contact with the dots.

For proximal-distal displacements, when a dot stimulus transfers from one finger to another, deciding whether the dot in question is indeed a single stationary dot is a problem which does not involve time directly. The question is: can the distinct paths on each finger be explained by a single dot? For instance, if the coalignment (in somatotopic coordinates) at the end-points of the two paths is sufficient, without formal definition, we can speculate that a single dot can account for these two paths. We can then further propose that similar notions are available in vision as expressed by the Gestalt laws of continuation and we can imagine further experiments to investigate this possibility.

The medial-lateral displacements give the brain a very different kind of problem. This type of stimuli trace on each finger paths that match perfectly in somatotopic coordinates: exactly the same paths that would be created by a single stationary dot. The difference that the interdigital displacements made is that the dot appeared too early or too late on the trailing finger with reference to the instant when the dot left the leading finger. These stimuli give the brain a space-time problem to solve where three quantities are intertwined, a time interval, a distance, and a speed. Here, our experiment can be related to paradigms used by vision researchers (Bridgeman and Stark, 1991; Ross et al., 1997) where subjects observed stationary or moving targets during saccadic movements. These observations can explain the greater asymmetry between different stimuli cases. For patterns 11 and 15, the dot were displaced in the same direction as the fingers moved and hence showed up too late but for patterns 9 and 13, it showed up too soon and the tolerance to mismatch is greater when the dots appears too late than can be predicted from the relative speed between a stationary dot and a moving finger. Also, pattern 12 and 16 correspond to the stationary condition of the pattern 11 and 15. This case is particularly interesting. The participants perceived a changing dot when it was stationary, which means that for the leftward displacements (stationary or moving condition), the participants based their answers on the first part of the pattern (the position of the first dot is always on the right for pattern $11,12,15,16$ ) and opted for a predictive strategy to give their answers.

The analogy with the visual system does not stop here since both modalities raise the question of reafference, which is central to the perception of immobility and movement (Wertheim, 1994). According to Wertheim, a more refined theory holds that the perception of an object's motion depends on the difference between its intrinsic movement and the detection threshold of the movement direction. Thus, during a smooth visual pursuit, motion is underestimated when the eye moves in the same direction as the stimulus since the differential velocity is small (Wertheim, 1994; Haarmeier and Thier, 1995). In the tactile domain, we could suppose the existence of a similar phenomenon. By analogy, for a stimulus moving in the same direction as the fingers, the speed is underestimated which may yield a perception of stationarity. Although our paradigm did not test explicitly for this hypothesis, our results agree with this possibility since our stimuli 11 and 15 could be interpreted as a dot having an apparent movement in the same direction as the finger, while stimuli 9 and 13 would be like a dot moving in the opposite direction.

\section{Postdictive and predictive strategies}

Our results are also connected to the question of how the brain deals with differential events (Cullen, 2004). When a percept, such as the stationary judgment of a moving dot, depends on events separated in time, the brain has two, not mutually exclusive strategies to process them. In the first, the postdictive strategy, the first event is kept in a memory trace to be compared with the second event in order to deduce, say, a distance or intensity differential. In the second, the predictive strategy, there is sufficient information in the first stimulus to predict the occurrence of the second. It is the prediction that is compared to the second event. Computer-controlled stimulus generation makes it possible to test for these possibilities (Bays et al., 2006a, 2006b). The asymmetry in our results in the case of the laterally displaced dots provides support for the predictive hypothesis. In effect, if the comparison was postdictive then whether the dot appears too soon or too late on the leading finger should not make a large difference. On the other hand, if the brain uses a predictive strategy, then a dot appearing too soon would be more detectable than a dot appearing too late, still satisfying the prediction but with some delay.

\section{Magnitude of the effect}

Returning to our initial motivation, which was to seek parallels, or lack thereof, between vision and touch, we 
may observe that the threshold for visual SSD is about $1^{\circ}$ (up to $4^{\circ}$, depending on delay and saccade amplitudes) whereas the angle subtended by the fovea is about $3-6^{\circ}$ (Weale, 1966). We found that displacement suppression in touch is of the order of $3 \mathrm{~mm}$ for small movements whereas the size of a tactile contact surface is about $10 \mathrm{~mm}$, therefore in a comparable ratio. On the other hand, the analogy breaks down if one considers that the visual fovea is surrounded by a large peripheral sensitive surface that has no equivalent in touch. Nevertheless, in both cases, the phenomenon of displacement suppression may be thought to be a mechanism contributing to the perception of a stable world. By analogy, engineers often design small amounts of hysteresis in systems in order to stabilize noisy or drifting signals, making them appear more stable than they really are. Displacement suppression is a form of hysteresis since the output (the percept) depends on the history of the inputs (selfgenerated movement plus stimulus external location) and not just on the inputs at one instant in time. Here, a change due to self-generated movement, call it "extracutaneous signals" by analogy with extra-retinal signals, suppresses the conscious awareness of a small change in the stimulus location.

In sum, the results of the experiment reported here provide the first evidence of a tactile suppression of displacement effect in the somatosensory modality, which is comparable to saccadic suppression of image displacement in the visual modality. In summary, we conclude that the effect actually yields two distinct interpretations. For proximal-distal displacements, corresponding to a geometrical Gestalt-like effect, asymmetrical results appear to be the result from the inhomogeneous tactile sensitivity of the fingertip. For lateral-medial displacements, asymmetrical results can be interpreted in terms of a time-space problem connected to reafference and differential processing strategies.

\section{Acknowledgments}

Special thanks to Dominique Aubert for help with the software. This research was supported by a Special Research Opportunity Grant from the Natural Sciences and Engineering Research Council of Canada (NSERC).

\section{References}

Bays PM, Husain, M (2007) Spatial remapping of the visual world across saccades. Neuroreport 18:12071213

Bays PM, Wolpert DM, Flanagan JR (2006a) Perception of the consequences of self-action is temporally tuned and event driven. Current Biology, 15:1125-1128

Bays PM, Flanagan JR, Wolpert DM (2006b) Attenuation of self-generated tactile sensations is predictive, not postdictive. PLoS Biol 4(2):e28

Bridgeman B, Hendry D, Stark L (1975) Failure to detect displacement of visual world during saccadic eye movements, Vision Research 15:719-722
Bridgeman B, Stark L (1991) Ocular proprioception and efference copy in registering visual direction. Vision Research 31:1903-1913

Chapman, CE (1994) Active versus passive touch: factors influencing the transmission of somatosensory signals to primary somatosensory cortex. Canadian Journal of Physiology and Pharmacology 72:558-570

Chapman, CE and Beauchamp, E (2006) Differential controls over tactile detection in humans by motor commands and peripheral reafference, Journal of Neurophysiology 96:1664-1675

Craig, JC (1999) Grating orientation as a measure of tactile spatial acuity. Somatosensory \& Motor Research 16:197-206

Cullen, KE (2004) Sensory signals during active versus passive movement. Current Opinion in Neurobiology 14:698-706

Deubel H, Schneider WX, Bridgeman B (2004) Different effects of eyelid blinking and target blanking on saccadic suppression of displacement. Perception \& Psychophysics 66:772-778

Dodge R (1900) Visual perception during eye movement, Psychological Review 7, 454-465

Dyhre-Poulson P (1978) Perception of tactile stimuli before ballistic and during tracking movements. In: G. Gordon (ed) Active touch, Pergamon Press, Oxford, pp 171-176

Fitzgerald PJ, Lane JW, Thakur PH, Hsiao SS (2006) Receptive field properties of the macaque second somatosensory cortex: representation of orientation on different finger pads. Journal of Neuroscience 26: 6473-6484.

Gallace A, Auvray M, Tan HZ, Spence C. (2006) When visual transients impair tactile change detection: a novel case of crossmodal change blindness?, Neuroscience Letters 398:280-285

Ghez C, Lenzi GL (1971) Modulation of sensory transmission in cat lemniscal system during voluntary movements, Pflügers Archiv European Journal of Physiology 323:273-278

Haarmeier T, Thier P (1996) Modification of the Filehne illusion by conditioning visual stimuli. Vision research, 36:741-750

Haggard P (2006) Sensory neuroscience: from skin to object in the somatosensory cortex. Current Biology 16: 884-886.

Hatwell Y (1986) Toucher l'espace. La main et la perception tactile de l'espace. Presses Universitaires de Lille, Lille

Heller MA (1986) Active and passive tactile braille recognition. Bulletin of the Psychonomic Society, 24:201-202

Johnson K O, Phillips JR (1981) Tactile spatial resolution. I. Two-point discrimination, gap detection, 
Authors' copy accepted to Experimental Brain Research, 2010.

grating resolution, and letter recognition, Journal of Neurophysiology, 46:1177-1191

Lenoir T (1994) Helmholtz and the materialities of communication, Osiris 9:185-88

Loomis JM (1985) Tactile recognition of raised characters: A parametric study, Bulletin of the Psychonomic Society, 23: 18-20

Matin E (1974) Saccadic suppression: A review and an analysis, Psychological Bulletin, 81, 899-917

Macmillan NA, Creelman CD (1991) Detection theory: A users guide. Cambridge press, Lawrence Erlbaum Associates, New York

Merleau-Ponty M (1945) Phénoménologie de la perception. Gallimard, Paris

Millar, S. (1997) Reading by Touch, Routledge, London

O'Regan, JK \& Noë A (2001) A sensorimotor account of vision and visual consciousness, Behavioral and Brain Sciences, 24:883-917

Poincaré H (1908) Science et Méthode, Flammarion, Paris

Rensink RA, O'Regan JK, Clark JJ (1987) To see or not to see: the need for attention to perceive changes in scenes, Psychological Science 8(5):368-373

Ross J, Morrone MC, Burr DC (1997) Compression of visual space before saccades. Nature 386:587-601

Stevenson SB, Volkmann FC, Kelly JP, Riggs LA (1986) Dependence of visual suppression on the amplitudes of saccades and blinks, Vision Research, 26:1815-1824.

Taylor-Clarke M, Jacobsen P, Haggard P (2004) Keeping the world a constant size: object constancy in human touch, Nature neuroscience, 7:219-220

Vitello MP, Ernst MO, Fritschi, M (2006) An instance of tactile suppression: Active exploration impairs tactile sensitivity for the direction of lateral movement. Proceedings of EuroHaptics 2006, pp 351-355

Vitevitch MS (2003) Change deafness: the inability to detect changes between two voices, Journal of Experimental Psychology: Human Perception Performance, 29:333-342

Volkmann FC, Riggs LA, Moore RK (1980) Eyeblinks and visual suppression, Science, 207:900-902.

Wallach H, Lewis C (1966) The effect of abnormal displacement of the retinal image during eye movements. Perception \& Psychophysics, 1:25-29.

Weale RA (1966) Why does the human retina possess a fovea? Nature 212:255-256

Wertheim AH (1994) Motion perception during selfmotion: The direct versus inferential controversy revisited. Behavioral and Brain Sciences: 17:293-355.

Williams SR, Shenesa J and Chapman CE (1998) Time course and magnitude of movement-related gating of tactile detection in humans. I. Importance of stimulus location, Journal of Neurophysiology, 79:947-963.
Final version Vol 206, No 3, pp. 299-310

Williams SR and Chapman CE (2000) time course and magnitude of movement-related gating of tactile detection in humans. II. Effect effects of stimulus intensity. Journal of Neurophysiology, 84:863-875.

Williams SR and Chapman CE (2002) Time course and magnitude of movement-related gating of tactile detection in humans. III. Effect of motor tasks, Journal of Neurophysiology, 88:1968-1979.

Wexler M, Panerai F, Lamouret I, Droulez J (2001) Selfmotion and the perception of stationary objects. Nature 409:85-88. 